\title{
Quasar astrophysics with the Space Interferometry Mission
}

\author{
S. C. Unwin ${ }^{1}$, A. E. Wehrle ${ }^{2}$, D. L. Meier ${ }^{1}$, \\ D. L. Jones ${ }^{1}$, and B. G. Piner ${ }^{3}$ \\ ${ }^{1}$ Jet Propulsion Laboratory, California Institute of Technology, 4800 Oak Grove Drive, \\ Pasadena, CA 91109; email: stephen.unwin@jpl.nasa.gov \\ ${ }^{2}$ Space Science Institute, 4750 Walnut Street, Suite 205, Boulder, CO 80301 \\ ${ }^{3}$ Whittier College, Dept. Physics \& Astronomy 13406 E. Philadelphia St., Whittier, CA 90608

\begin{abstract}
Optical astrometry of quasars and active galaxies can provide key information on the spatial distribution and variability of emission in compact nuclei. The Space Interferometry Mission (SIM PlanetQuest) will have the sensitivity to measure a significant number of quasar positions at the microarcsecond level. SIM will be very sensitive to astrometric shifts for objects as faint as $V=19$. A variety of AGN phenomena are expected to be visible to SIM on these scales, including time and spectral dependence in position offsets between accretion disk and jet emission. These represent unique data on the spatial distribution and time dependence of quasar emission. It will also probe the use of quasar nuclei as fundamental astrometric references. Comparisons between the time-dependent optical photocenter position and VLBI radio images will provide further insight into the jet emission mechanism. Observations will be tailored to each specific target and science question. SIM will be able to distinguish spatially between jet and accretion disk emission; and it can observe the cores of galaxies potentially harboring binary supermassive black holes resulting from mergers.
\end{abstract}

Keywords. astrometry, instrumentation: interferometers, quasars: general, radiation mechanisms: general

Astrometry at microarcsecond accuracy is a new probe of the central engine of quasars. With flexible scheduling and single-measurement accuracy of 10-15 $\mu$ as, SIM (see Unwin et al. 2007) is an ideal instrument to coordinate with ground instruments (optical monitoring and VLBI imaging) and space instruments (IR and X-ray) to monitor positions of blazars during outbursts. Position shifts of variable quasar nuclei relative to a local reference frame are expected, even though they will not be spatially resolved by the SIM interferometer. Specific questions that can be probed, using observations of a small number of highly variable objects, include the following: What are the sizes and geometric relations between the components of the core region (jets, accretion disk, hot corona)? How are galaxy mergers related to the AGN phenomenon - do binary black holes result from mergers and how common are they? Does the most compact non-thermal optical emission from an AGN come from an accretion disk or from a relativistic jet? Does the separation of the radio core and optical photocenter of the quasars used for the reference frame tie change on the timescales of their photometric variability, or is the separation stable? Do the cores of galaxies harbor binary supermassive black holes remaining from galaxy mergers?

In radio-loud quasars, powerful (and often relativistic) jets are ejected from the central engine. From jet models, e.g., Königl (1981) we can estimate that there will be astrometric offsets between radio and optical (up to about $70 \mu \mathrm{as}$, using the quasar 3C 345 at $z=0.6$ as an example), and across the optical band (up to $30 \mu \mathrm{as}$ ). Figure 1 shows a sketch of the region where jet acceleration begins, showing the various physical components. SIM can 
probe the sub-parsec structure using time-dependent and color-dependent astrometry. The blue accretion disk and red corona are very compact and spatially coincident, so SIM would not expect to detect time- or color-dependent astrometric shifts in these components. However, if the red jet dominates, then a color shift should be seen, with the shift aligned with the jet, and any astrometric variability should also be co-aligned.
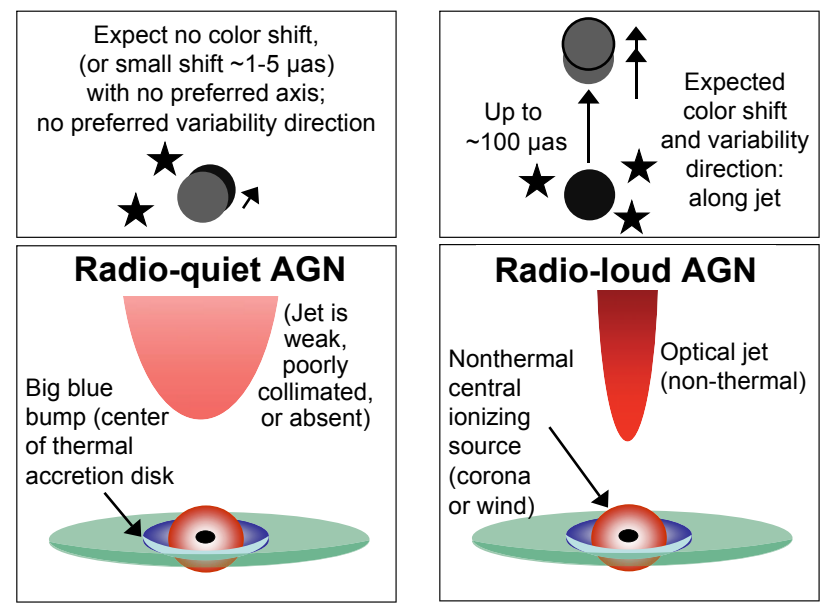

Figure 1. Schematic diagram of the structure of a typical quasar on scales $\lesssim 1$ pc. The lower left panel illustrates a radio-quiet AGN where the emission is dominated by the corona and/or accretion disk. As shown by the upper left panel, variability is not expected to be manifested as an astrometric shift, nor would the shift be color-dependent. For a radio-loud AGN or a blazar (right panels) variability (which may be color dependent) in the jet will cause a shift in photocenter which is aligned with the jet axis.

SIM can also search for evidence of binary black holes which may be end-products of galaxy mergers. If massive binary black holes are found, we have a new means of directly measuring the masses and estimating the coalescence lifetimes of the binaries. One promising candidate is OJ $287(z=0.3)$, based on brightness variations with 12-year periodicity Kidger (2000), Valtonen et al. (2006). About $14 \mu$ as of orbital motion may be expected during a five-year span.

\section{Acknowledgements}

The authors would like to thank their many colleagues, too numerous to mention, whose vision and determination over the course of more than a decade have brought SIM PlanetQuest to its current mature design. Through their efforts, we are now poised, technology in hand, to build the next generation of astrophysics instruments based on interferometry. The research described in this paper was carried out at the Jet Propulsion Laboratory, California Institute of Technology, under contract with the National Aeronautics and Space Administration.

\section{References}

Kidger, M. 2000, $A J, 119,2053$

Königl, A. 1981, ApJ, 243, 700

Unwin, S. C., et al. 2007, PASP, in press

Valtonen, M., et al. 2006, ApJ (Letters), 6423, L9 